Internist $2021 \cdot 62: 928-936$

https://doi.org/10.1007/s00108-021-01120-y

Angenommen: 19. Juli 2021

Online publiziert: 12. August 2021

(c) Springer Medizin Verlag $\mathrm{GmbH}$, ein Teil von

Springer Nature 2021

Redaktion

E. Märker-Hermann, Wiesbaden

D. Nowak, München

\section{Die parallele Pandemie?}

\author{
Maßnahmen zur Verbesserung der Arbeits- und \\ Beanspruchungssituation bei Personal in der \\ Krankenversorgung
}

\author{
Matthias Weigl ${ }^{1,2} \cdot$ Julia Schreyer ${ }^{2}$ \\ 'Institut für Patientensicherheit, Medizinische Fakultät, Universität Bonn, Bonn, Deutschland \\ ${ }^{2}$ Institut und Poliklinik für Arbeits-, Sozial- und Umweltmedizin, Klinikum der Universität München, \\ München, Deutschland
}

\section{Zusammenfassung}

\section{In diesem Beitrag}

- Verbesserung der Arbeits- und Gesundheitssituation des Gesundheitspersonals

- Evidenzlage zu Interventionsansätzen für die Gesundheitsförderung bei Gesundheitspersonal

- Gibt es effektive Interventionen zur Förderung der psychischen Gesundheit des Personals während COVID-19?

- Rahmenbedingungen für erfolgreiche Veränderungen

Die Gesundheit und Leistungsfähigkeit der Beschäftigten ist essenziell für ein funktionierendes Gesundheitswesen. Ein substanzieller Anteil der Beschäftigten berichtet - auch schon vor der Coronavirus-disease-2019(COVID-19)-Pandemie Beeinträchtigungen der psychischen Gesundheit in der Arbeit. Dieser Beitrag umreißt Wissensstand und Evidenz zu Fördermaßnahmen der psychischen Gesundheit am Arbeitsplatz mit besonderem Schwerpunkt auf der Arbeits- und Tätigkeitsgestaltung. Neben einer ersten Übersicht zu Ansätzen werden auch Faktoren erfolgreicher und effektiver Ansätze angesprochen.

\section{Schlüsselwörter}

COVID-19 · Psychische Gesundheit · Arbeitsmedizin · Beruflicher Stress · Interventionen am Arbeitsplatz

\section{Hintergrund}

Schon vor und erst recht durch die "coronavirus disease 2019“ (COVID-19) hatten und haben wir - provokant formuliert eine zweite Pandemie: Ein erheblicher Anteil des im Gesundheitswesen tätigen Personals berichtet kritische Werte von Erschöpfung, Burn-out, Depression, Angst und Unzufriedenheit [1, 2]. Eine Übersichtsarbeit über 100 Einzelstudien resümiert, dass jede*r Vierte in der COVID-19Versorgung tätige Beschäftigte kritische Ängstlichkeits- und Depressionswerte aufwies [3]. Der Anteil Beschäftigter mit psychischen Fehlbeanspruchungen war auch schon vor der Pandemie beträchtlich [4, 5]. Das trifft auch für Untersuchungen in Deutschland zu (beispielsweise [6]). Es gilt also, dieser parallelen Pandemie mit effektiven Maßnahmen zur Förderung der Gesundheit des Personals entgegenzutreten [7-9].

Die Qualität der Arbeit im Krankenhaus und der Arztpraxis ist mitverantwortlich für die hohe Rate an gesundheitlichen und psychischen Fehlbeanspruchun- gen des Gesundheitspersonals. Schlechte strukturelle und organisatorische Rahmenbedingungen sowie Belastungen durch die Tätigkeit sind ein zentraler Einflussfaktor für die Gesundheit des Personals. Das war vor der Pandemie so und die Studien zur Gesundheitssituation während der COVID19-Pandemie bestätigen dies [1, 9-11].

\section{\) Die COVID-19-Pandemie hat die Probleme und Belastungsfaktoren in der Patientenversorgung akzentuiert}

Die COVID-19-Pandemie hat die bisherigen, zuweilen nur latent vorhandenen oder ungenügend benannten Probleme und Belastungsfaktoren in der Patientenversorgung akzentuiert [7]. In D Tab. 1 haben wir nach unsystematischer Recherche die aus unserer Sicht zentralen Belastungsfaktoren zusammengetragen, die in der Literatur für die kritische Gesundheits- und Beanspruchungssituation aufgeführt werden.

Die Validität und methodische Qualität vieler Studien zu pandemiespezifischen 


\begin{tabular}{|c|c|c|}
\hline Bereich & $\begin{array}{l}\text { Generische Belastungsfaktoren (vor, während und nach } \\
\text { COVID-19 }[6,12-14] \text { ) }\end{array}$ & $\begin{array}{l}\text { Pandemiebezogene Belastungsfaktoren, spezifisch für } \\
\text { COVID-19-Versorgung }[1,2,9,11,15-17]\end{array}$ \\
\hline \multirow[t]{4}{*}{ Gesellschaft } & Nachwuchs- und Personalmangel in Pflegeberufen & Zeitliche Perspektive \\
\hline & Anerkennung von Pflegeberufen & Stigmatisierung und Diskriminierung \\
\hline & \multirow[t]{2}{*}{ Hohe Erwartungen } & Angst vor Kollaps \\
\hline & & Diffuse Panik und Lockdowns \\
\hline \multirow[t]{2}{*}{ Gesundheitssystem } & Bürokratische Regelungen und Anforderungen & $\begin{array}{l}\text { Direkte Exposition gegenüber COVID-19-Patient*innen in } \\
\text { der Versorgung }\end{array}$ \\
\hline & Grundfinanzierung & Erhöhter Versorgungsaufwand \\
\hline \multirow[t]{5}{*}{ Organisation } & Hohe Arbeitsbelastung/hoher Zeitdruck & Unterbesetzung \\
\hline & Personelle Ausstattung & Überstunden \\
\hline & Hierarchien und autoritäre Führungskultur & Unklare Kommunikation \\
\hline & Rollenunklarheiten & \multirow[t]{2}{*}{ Mangelnde Ausstattung mit persönlicher Schutzausrüstung } \\
\hline & Arbeit in Schichtdienst und unkonventionelle Arbeitszeiten & \\
\hline \multirow[t]{2}{*}{ Team } & Probleme der Führung & Mangel an praktischer Unterstützung \\
\hline & Abstimmungsprobleme im Team und zwischen Bereichen & Mangelnde Erholungsmöglichkeiten durch Personalmangel \\
\hline \multirow[t]{4}{*}{ Individuell } & Mangelnde Möglichkeiten für Pausen und Erholung & $\begin{array}{l}\text { Sorge um eigene Infektion sowie von Familie und Angehöri- } \\
\text { gen }\end{array}$ \\
\hline & $\begin{array}{l}\text { Erlebtes Ungleichgewicht zwischen Aufwand und Ertrag } \\
\text { (Gratifikationskrise) }\end{array}$ & $\begin{array}{l}\text { Hilflosigkeit, Kontrollverlust - moralische Zweifel und Ge- } \\
\text { fühle der Ohnmacht }\end{array}$ \\
\hline & $\begin{array}{l}\text { Unvereinbarkeit von außerberuflichen Belastungen und } \\
\text { Arbeitsanforderungen }\end{array}$ & Mangelnde Arbeitserfahrung \\
\hline & $\begin{array}{l}\text { Angst vor Behandlungs- und Kunstfehlern und den juristi- } \\
\text { schen Folgen }\end{array}$ & Angst vor Stigmatisierung \\
\hline \multirow[t]{6}{*}{ Arbeitsaufgabe } & Umgang mit schwierigen Fällen & \multirow{6}{*}{$\begin{array}{l}\text { Kontakt mit hochinfektiösen Patient*innen (ggf. ohne Ab- } \\
\text { klärung) }\end{array}$} \\
\hline & Erleben von Tod und Sterben & \\
\hline & Limitierte Autonomie in der Tätigkeit & \\
\hline & Hohe Verantwortung & \\
\hline & Körperliche Belastungen & \\
\hline & Mangelnde Rückmeldung & \\
\hline
\end{tabular}

Belastungen wie auch die Gültigkeit für verschiedene klinische Bereiche wird kritisch beurteilt [1, 15]. Gleichwohl zeigen die Studien, dass die COVID-19-Pandemie viele Grundprobleme und strukturbedingte Belastungen nicht verändert hat.

\section{Verbesserung der Arbeits- und Gesundheitssituation des Gesundheitspersonals}

Maßnahmen zur Verbesserung der Arbeitssituation und mithin der Gesundheit sind Teil eines mitarbeiterorientierten Arbeitsund Gesundheitsschutzes und imperativ für die Aufrechterhaltung der Leistungsfähigkeit und letztlich des gesamten Versorgungssystems. Im Kontrast zu der immensen Menge an beschreibenden Prävalenz- und Beobachtungsstudien zur Arbeits- und Gesundheitssituation von Gesundheitspersonal sind Interventionsstu- dien sowie systematische Übersichtsarbeiten nach wie vor Mangelware. Anzahl und Qualität der Studien zur Förderung der Gesundheit des Personals in Krankenhäusern und stationären Pflegeeinrichtungen nehmen zu, die Evidenzlage ist jedoch inkonsistent [18-24].

In der Gesamtschau wird oft eine Unterscheidung zwischen verhaltens- oder verhältnisorientierten und kombinierten Interventionsansätzen getroffen [25]:

- Verhaltensorientierte Ansätze zielen auf die individuellen Beschäftigten und deren individuelle Situation, deren Erleben und Verhalten sowie deren (Stress-)Reaktionen. Oftmals geht es hier um eine Modifikation oder Anpassung von Verhaltensweisen (Coping) im Umgang mit stressintensiven Phasen oder Belastungen (beispielsweise durch das Angebot von Yoga, Stress-, Achtsamkeits- oder Resilienztrainings).
- Verhältnisorientierte Maßnahmen zielen auf eine Änderung der Umgebungs- und Tätigkeitsbedingungen, um umgebungs- und strukturbedingte Risiken für Gesundheit und Wohlbefinden zu minimieren [26-28]. Im besten Fall zielen sie auf die Gestaltung von leistungs-, gesundheits- und insbesondere auch persönlichkeitsförderlichen Bedingungen am Arbeitsplatz [26, 28, 29].

- Kombinierte Ansätze tragen oft der Komplexität Rechnung und zielen auf die gleichzeitige und einheitliche Änderung ungünstiger Umgebungsbedingungen sowie individueller Verhaltens- oder Erlebensweisen. 
Hier steht eine Anzeige.

黑 Springer 
Hier steht eine Anzeige.

黑 Springer 


\section{Evidenzlage zu Interventionsan- sätzen für die Gesundheitsförde- rung bei Gesundheitspersonal}

Die Literatur zu Interventionen ist limitiert. Das gilt insbesondere für systematische und professionsübergreifende Untersuchungen zur Effektivität von organisationalen und strukturellen Maßnahmen [18, 19]. Umfangreicher ist hingegen die Literaturbasis für verhaltensorientierte Studien und Trainings.

- Ein Cochrane-Review zu Arbeitsstressinterventionen bei Gesundheitspersonal schloss insgesamt 20 organisationale Interventionen ein und stellte dort lediglich für den Teilbereich Dienstzeiten („work schedules") einen positiven Effekt fest (beispielsweise Einführung von Erholungstagen an Wochenenden oder kürzere Blöcke aufeinanderfolgender Dienste). Auf Basis der 41 eingeschlossenen individuumsbezogenen Interventionen zeigte sich ein positiver Effekt für Entspannungsinterventionen und kognitiv-behaviorale Ansätze, wobei die Autor*innen die Qualität der Studien als gering bewerten [30].

- Eine Übersichtsarbeit zu Burn-outInterventionen bei Ärzt*innen zeigte, dass organisationale Interventionen $(n=8)$ einen höheren Effekt aufwiesen als individuumsorientierte Ansätze wie Achtsamkeitstrainings oder kognitivbehaviorale Trainings [19].

- Ein weiterer Review zu Burn-out bei Ärzt*innen wies keine Vorteile der (wiederum wenigen) organisationalstrukturellen Gestaltungsmaßnahmen gegenüber individuumsorientierten Ansätzen auf [18]. Diese Studien bezogen sich auf Arbeitszeitreformen bei Ärzt*innen, Schichtplanänderungen und Änderungen klinischer Praktiken.

- Eine weitere Cochrane-Arbeit zeigte, dass Interventionen zur Stärkung der individuellen Resilienz einen moderaten Effekt auf Stress und Stresswahrnehmung und einen kleinen Effekt auf das Depressionsniveau von Gesundheitspersonal haben [31].

- Eine Übersichtsarbeit mit Metaanalyse von 4 Übersichtsarbeiten zu Burnout bei Ärzt*innen kommt zu dem Schluss, dass weder für organisationale noch individuelle Ansätze bislang eine deutliche Befundlage vorliegt und es angesichts der heterogenen Studienlage insbesondere auf die Rahmenbedingungen und Voraussetzungen erfolgreicher bzw. nichterfolgreicher Gestaltungsansätze ankommt [32].

Im Bereich organisationaler Interventionen der Gesundheitsförderung wurden verschiedenste Gestaltungsansätze berichtet, etwa Reorganisation von Dienstplänen und Reduktion von Arbeitszeit oder der Arbeitsmenge, Förderung von Teamwork, Anpassungen in der Arbeitsorganisation und Schnittstellengestaltung, Redefinition von Rollenerwartungen, Behebung von Problemen der Zusammenarbeit sowie erweiterte Beteiligung bei Entscheidungen [19, 33]. Spezifisch für den ärztlichen Bereich werden organisationale Verbesserungsansätze insbesondere für folgende Themen und Probleme vorgeschlagen: Ungleichgewicht von Arbeitsmenge und Arbeitszeit, ungenügende Autonomie und mangelnde Entscheidungsmöglichkeiten in der Patientenversorgung, ungenügende soziale Unterstützung am Arbeitsplatz, mangelnde Anerkennung der Arbeit angesichts anziehender finanzieller Vorgaben und Restriktionen, mangelnde organisationale Gerechtigkeit sowie divergierende Werthaltung von Ärzt*innen und den sie beschäftigenden Organisationen [34, 35].

\section{॥ Erfolgreiche Maßnahmen nutzen reflektive und mitarbeiterorientierte Verbesserungspraktiken}

In den vergangenen Jahren wurden auch verschiedene Interventionen untersucht, die sich vornehmlich der Stärkung des sozialen Austauschs untereinander und der kollegialen Unterstützung bedienen. Im angloamerikanischen Raum sind hier insbesondere sogenannte "Schwartz rounds" etabliert, in denen interprofessionelle Teams insbesondere ihr Erleben emotionaler und stressverursachender Situationen reflektieren [36]. Aufmerksamkeit haben auch Ansätze erhalten, in denen Führungskräfte systematische VorOrt-Visitationen durchführen („positive leadership walk rounds"), um spezifische Arbeitsprobleme der Beschäftigten kennenzulernen und zu beheben [37]. Gesundheits- oder Qualitätszirkel unter aktiver Partizipation des Personals sind hier eine Kombination von reflektiven und lösungsorientierten Verbesserungspraktiken $[38,39]$.

\section{Gibt es effektive Interventionen zur Förderung der psychischen Gesundheit des Personals während COVID-19?}

Studien zu effektiven Interventionen sowie deren Evidenz sind begrenzt. Es bestehen mehrere Probleme der internen und externen Validität, unter anderem gibt es wenige kontrollierte Interventionsstudien, kaum Abgleich zur präpandemischen Situation und einen Überhang von individuums- gegenüber verhältnispräventiven Angeboten [15, 40, 41]. Daher sind die berichteten Erkenntnisse vorläufig, können jedoch auch in Bezug zu früheren Pandemiegeschehen gebracht werden [16, 42]. Insgesamt lassen sich die berichteten Interventionen zur Förderung der psychischen Gesundheit während der COVID-19-Pandemie in drei Gruppen aufgliedern [15, 16, 41]:

- Organisationsbezogene Maßnahmen:

- Unterstützungsangebote, z. B. Helplines

- Unterstützung von Beschäftigten in häuslicher Quarantäne

- Positives Feedback

- Klare Kommunikation

- Stringente und effektive Infektionsschutzmaßnahmen

- Anpassung von Versorgungsstrategien und -prozeduren bei hochinfektiösen Patient*innen

- Arbeits-/Schichtplangestaltung

- Team- und soziale Unterstützungsmaßnahmen:

- Ausreichende Unterstützungsangebote

- Genügende Pausen- und Erholungszeiten, auch zwischen den Schichten

- Kollegiale Unterstützung

- Anerkennung und Wertschätzung

- Personenbezogene Maßnahmen:

- Trainingsangebote zur Kompetenzentwicklung

- Ausreichende Zeiten für Erholung und Regeneration 
- Bedarfsorientierte psychosoziale Unterstützung

- Psychologische und psychotherapeutische Beratungs- und Unterstützungsangebote zu Erhalt oder Stärkung psychologischer Resilienz

Die bisherig berichteten Erfahrungen lassen vermuten, dass die Grundzüge der effektiven Intervention zur Stressreduktion und Gesundheitsförderung beim Personal ähnlichen Grundprinzipien und Wirkweisen konventioneller Ansätze entsprechen; wie kontinuierliche Befragungen, zeitliche Freiräume zum Lernen und sichere Lernumgebungen [41, 43, 44]. Zentral ist gerade die Passung der Unterstützungs- und Interventionsangebote zu akutem Bedarf: Was hilft das Angebot einer Videokonsultation mit einer Psycholog*in, wenn es an persönlicher Schutzausrüstung im Bereich fehlt [15]?

\section{>) Organisationale und tätigkeits- immanente Belastungsfaktoren am Arbeitsplatz sollten adressiert werden}

Zudem berichten mehrere Studien, dass viele Beschäftigte individuelle Strategien (gerade auch außerhalb des Arbeitsplatzes) anwendeten, um mit der Belastungssituation fertigzuwerden [15]. Auch ist die Akzeptanz zusätzlicher Angebote (wie Stress- oder Resilienztrainings) eher begrenzt und es sollten eher organisationale und tätigkeitsimmanente Belastungsfaktoren am Arbeitsplatz adressiert werden $[15,42]$.

\section{Rahmenbedingungen für erfolgreiche Veränderungen}

Für den Erfolg von Gesundheitsförderungsmaßnahmen im Gesundheitswesen werden verschiedene strukturelle und prozessbezogene Faktoren als kritisch genannt:

- Systemische Veränderungen, für die sich insbesondere auf organisationaler und Makroebene effektive Ansätze finden:

- Auf den obersten Leitungsebenen, in Führungs- und Kontrollgremien: proaktives Bereitstellen von Ressourcen und die in allen Entscheidungen immanente Rücksichtnahme auf die Gesundheit von Beschäftigten [7] - psychische Gesundheit als Bestandteil der Organisationskultur [24]

- Auch in den Fachverbänden ein Bewusstsein mitsamt Aktivitäten für die Gesundheit ihrer Mitglieder (beispielsweise vergleichbar mit der Initiative der US-amerikanischen Nationalen Akademie für Medizin „Action Collaborative on Clinician Well-Being and Resilience")

- Partizipative Ansätze: aktive Teilhabe des betroffenen Personals bei der Identifikation belastender Arbeitsbedingungen, Wahl und Entwicklung geeigneter Verbesserungsansätze sowie Implementierung dieser Lösungen [45]. Die Methode der sogenannten (interprofessionellen) Gesundheitszirkel ist hier eine praktisch sehr gut handhabbare Vorgehensweise $[39,46]$.

- Messung und Überprüfung: Prüfen der Interventionseffekte durch wiederholtes Messen der Zufriedenheit und psychischen Gesundheit des Personals (beispielsweise durch kurze Umfragen oder konsequente Gefährdungsbeurteilung psychischer Belastungen), insbesondere in Phasen akuter Belastungen [1]

- Lokale Adaptation: Maßnahmen sollten an spezifische Gegebenheiten und Bedarfe vor Ort angepasst werden sowie vorhandene Ressourcen und zeitliche Bedingungen berücksichtigen und damit den verschiedenen Einflussebenen Rechnung tragen [27, 47-49].

- Komplexität anerkennen: Verschiedene Faktoren auf Ebene des Individuums, der Organisation und der Gesellschaft beeinflussen die mentale Gesundheit; eine Intervention muss dieser Komplexität gerecht werden und die Diversität innerhalb der Angestelltengruppen bezüglich individueller Bedürfnisse, Herausforderungen und Möglichkeiten berücksichtigen [24].

- Ressourcen schaffen: Die Teilnahme an Interventionen wird durch fehlende Zeit, Ressourcen sowie mangelnde Information oder Kommunikation zu den Aktivitäten erschwert [21];
Ressourcen sollten durch Führung und Management gewährleistet sein [24].

\section{Fazit für die Praxis}

- Mangelnde psychische Gesundheit des Personals ist ein Indikator für systemische Missstände.

- Günstige Rahmenbedingungen am Arbeitsplatz fördern die Zufriedenheit des Personals sowie die Qualität der Versorgung.

- Gesunde und leistungsfähige Beschäftigte im Gesundheitswesen gewährleisten eine effektive und qualitativ hochwertige Versorgung der Patient*innen.

- In Anbetracht der zunehmenden Evidenz effektiver Interventionsmaßnahmen gilt es, günstige Arbeitsbedingungen durch Maßnahmen der Arbeitsgestaltung, Personal- und Organisationsentwicklung zu etablieren, unabhängig von dieser oder der nächsten Pandemie.

Korrespondenzadresse

\section{Prof. Matthias Weigl}

Institut für Patientensicherheit, Medizinische Fakultät, Universität Bonn Gebäude 02 (Auenbruggerhaus), VenusbergCampus 1, 53127 Bonn, Deutschland matthias.weigl@ukbonn.de

\section{Einhaltung ethischer Richtlinien}

Interessenkonflikt. M. Weigl und J. Schreyer geben an, dass kein Interessenkonflikt besteht.

Für diesen Beitrag wurden von den Autoren keine Studien an Menschen oder Tieren durchgeführt. Für die aufgeführten Studien gelten die jeweils dort angegebenen ethischen Richtlinien.

\section{Literatur}

1. Sanghera J, Pattani N, Hashmi Y, Varley KF, Cheruvu MS, Bradley A et al (2020) The impact of SARS-coV-2 on the mental health of healthcare workers in a hospital setting - a systematic review. J Occup Health 62(1):e12175. https://doi.org/10. 1002/1348-9585.12175

2. Morawa E, Schug C, Geiser F, Beschoner P, JergBretzke L, Albus C et al (2021) Psychosocial burden and working conditions during the COVID19 pandemic in Germany: the VOICE survey among 3678 health care workers in hospitals. J Psychosom Res 144:110415. https://doi.org/10. 1016/j.jpsychores.2021.110415

3. Sahebi A, Nejati-Zarnaqi B, Moayedi S, Yousefi K, Torres M, Golitaleb M (2021) The prevalence of anxiety and depression among healthcare workers during the COVID-19 pandemic: an umbrella review of meta-analyses. Prog Neuropsychopharmacol Biol Psychiatry 107:110247. https://doi.org/ 10.1016/j.pnpbp.2021.110247 
4. van Mol MMC, Kompanje EJO, Benoit DD, Bakker J, Nijkamp MD (2015) The prevalence of compassion fatigue and burnout among healthcare professionals in intensive care units: a systematic review. PLoS ONE 10(8):e136955. https://doi.org/10.1371/journal.pone. 0136955

5. Rotenstein LS, Torre M, Ramos MA, Rosales RC, Guille C, Sen S et al (2018) Prevalence of burnout among physicians: a systematic review. JAMA 320(11):1131-1150. https://doi.org/10.1001/ jama.2018.12777

6. Beschoner P, Limbrecht-Ecklundt K, Jerg-Bretzke L (2019) Psychische Gesundheit von Ärzten. Nervenarzt 90(9):961-974. https://doi.org/10.1007/ s00115-019-0739-x

7. Dzau VJ, Kirch D, Nasca T (2020) Preventing a parallel pandemic - a national strategy to protect clinicians' well-being. N Engl J Med 383(6):513-515. https://doi.org/10.1056/NEJMp2011027

8. Krystal JH (2020) Responding to the hidden pandemic for healthcare workers: stress. Nat Med 26(5):639. https://doi.org/10.1038/s41591-0200878-4

9. Bendau A, Ströhle A, Petzold MB (2021) Mental health in health professionals in the COVID19 pandemic. In: Rezaei N (Hrsg) Coronavirus disease-COVID-19. Springer, Cham, S737-757

10. Bohlken J, Schömig F, Lemke MR, Pumberger $M$, Riedel-Heller SG (2020) COVID-19-Pandemie: Belastungen des medizinischen Personals. Psychiatr Prax 47(4):190-197. https://doi.org/10.1055/a1159-5551

11. Schulze S, Holmberg C (2021) Bedeutung und Belastung von Pflegekräften während der CoronaKrise. Public Health Forum 29(1):32-35. https:// doi.org/10.1515/pubhef-2020-0114

12. Kinman G, Teoh K (2018) What could make a difference to the mental health of UK doctors? A review of the research evidence

13. Lee RT, Seo B, Hladkyj S, Lovell BL, Schwartzmann L (2013) Correlates of physician burnout across regions and specialties: a meta-analysis. Hum Resour Health 11:48. https://doi.org/10.1186/ 1478-4491-11-48

14. Weigl M (2014) Beschäftigte im Ärztlichen Dienst. In: Angerer $\mathrm{P}$, Glaser J, Gündel H, Henningsen $\mathrm{P}$, Lahmann C, Letzel S et al. (Hrsg) Psychische und psychosomatische Gesundheit in der Arbeit Wissenschaft, Erfahrungen, Lösungen aus Arbeitsmedizin, Arbeitspsychologie und psychosomatischer Medizin. Ecomed Medizin, Landsberg

15. Muller AE, Hafstad EV, Himmels JPW, Smedslund G, Flottorp S, Stensland $S \varnothing$ et al (2020) The mental health impact of the covid-19 pandemic on healthcare workers, and interventions to help them: a rapid systematic review. Psychiatry Res 293:113441. https://doi.org/10.1016/j.psychres. 2020.113441

16. Cabarkapa S, Nadjidai SE, Murgier J, Ng CH (2020) The psychological impact of COVID-19 and other viral epidemics on frontline healthcare workers and ways to address it: a rapid systematic review. Brain Behav Immun Health 8:100144. https://doi. org/10.1016/j.bbih.2020.100144

17. Zerbini $G$, Ebigbo A, Reicherts $P$, Kunz $M$, Messman H (2020) Psychosocial burden of healthcare professionals in times of COVID19-a survey conducted at the University Hospital Augsburg. Ger Med Sci 18:Doc5. https://doi.org/ 10.3205/000281

18. West CP, Dyrbye LN, Erwin PJ, Shanafelt TD (2016) Interventions to prevent and reduce physician burnout: a systematic review and meta-analysis.

\section{Is this the parallel pandemic? Measures to improve working conditions and stress levels among health care personnel}

Employee health and ability to perform is essential to a functioning health care system. Even before the coronavirus disease 2019 (COVID-19) pandemic, a substantial proportion of employees reported impaired mental health at work. This paper outlines the state of knowledge and evidence on interventions to promote mental health in the workplace, with particular focus on the organization of work and activities. In addition to an initial review of approaches, the factors facilitating successful and effective approaches are addressed.

\section{Keywords}

COVID-19 - Mental health · Occupational medicine · Occupational stress · Interventions at work

Lancet 388(10057):2272-2281. https://doi.org/ 10.1016/s0140-6736(16)31279-x

19. Panagioti M, Panagopoulou E, Bower P, Lewith $G$, Kontopantelis E, Chew-Graham C et al (2017) Controlled interventions to reduce burnout in physicians: a systematic review and meta-analysis. JAMA Intern Med 177(2):195-205. https://doi.org/ 10.1001/jamainternmed.2016.7674

20. Nowrouzi B, Lightfoot N, Lariviere M, Carter L, Rukholm E, Schinke R et al (2015) Occupational stress management and burnout interventions in nursing and their implications for healthy work environments: a literature review. Workplace Health Saf 63(7):308-315. https://doi.org/10. 1177/2165079915576931

21. Brand SL, Thompson Coon J, Fleming LE, Carroll L, Bethel A, WyattK (2017) Whole-system approaches to improving the health and wellbeing of healthcare workers: a systematic review. PLoS ONE 12(12):e188418. https://doi.org/10.1371/journal. pone. 0188418

22. Ruotsalainen J, Verbeek J, Mariné A, Serra C (2015) Preventing occupational stress in healthcare workers. Cochrane Database Syst Rev. https://doi. org/10.1002/14651858.CD002892.pub5

23. Weigl M (2019) Organisationale Interventionen zur Verbesserung der Arbeitsbedingungen im Krankenhaus. In: Angerer P, Gündel H, Brandenburg GW, Nienhaus A, Letzel S, Nowak D (Hrsg) Arbeiten im Gesundheitswesen: Psychosoziale Arbeitsbedingungen-Gesundheit der Beschäftigten - Qualität der Patientenversorgung. ecomed Medizin, Landsberg, $S 301$

24. Gray P, Senabe S, Naicker N, Kgalamono S, Yassi A, Spiegel JM (2019) Workplace-based organizational interventions promoting mental health and happiness among healthcare workers: a realist review. Int J Environ Res Public Health. https://doi.org/10.3390/ijerph16224396

25. Lamontagne AD, Keegel T, Louie AM, Ostry A, Landsbergis PA (2007) A systematic review of the job-stress intervention evaluation literature, 1990-2005. Int J Occup Environ Health 13(3):268-280. https://doi.org/10.1179/oeh. 2007.13.3.268

26. Nielsen K, Noblet A (2018) Organizational interventions for health and well-being: a handbookfor evidence-based practice. Routledge,

27. Glaser J, Weigl M (2011) Verschreibungsfähig, trotz Risiken und Nebenwirkungen : Arbeitspsychologische Organisationsentwicklung im Krankenhaus und Altenpflegeheim. Z Organisationsentwicklung 30(2):11-19
28. Ulich E (Hrsg) (2003) Arbeitspsychologie in Krankenhaus und Arztpraxis: Arbeitsbedingungen, Belastungen, Ressourcen. Huber, Bern

29. Ulich E, Wülser M (2012) Gesundheitsmanagement in Unternehmen: Arbeitspsychologische Perspektiven, 5. Aufl. Springer, Wiesbaden

30. Ruotsalainen $\mathrm{JH}$, Verbeek $\mathrm{JH}$, Marine A, Serra C (2015) Preventing occupational stress in healthcare workers. Cochrane Database Syst Rev 4:Cd2892. https://doi.org/10.1002/14651858. CD002892.pub5

31. Kunzler AM, Helmreich I, Chmitorz A, König J, Binder H, Wessa M et al (2020) Psychological interventions to foster resilience in healthcare professionals. CochraneDatabase Syst Rev.https:// doi.org/10.1002/14651858.CD012527.pub2

32. Kalani SD, Azadfallah P, Oreyzi H, Adibi P (2018) Interventions for physician burnout: a systematic review of systematic reviews. Int J Prev Med 9:81. https://doi.org/10.4103/ijpvm.JJPVM 255 18

33. Swensen SJ, Shanafelt T (2017) An organizational framework to reduce professional burnout and bring back joy in practice. Jt Comm J Qual Patient Saf 43(6):308-313

34. Brower KJ (2017) Organization-level interventions to promote physician health and well-being: from taking care of physicians to giving care to patients. Physician mental health and well-being. Springer, S295-323

35. Olson K, Marchalik D, Farley H, Dean SM, LawrenceEC, HamidiMSetal (2019) Organizational strategies to reduce physician burnout and improve professional fulfillment.Curr Probl Pediatr Adolesc Health Care 49(12):100664. https://doi. org/10.1016/j.cppeds.2019.100664

36. Flanagan E, Chadwick R, Goodrich J, Ford C, Wickens R (2020) Reflection for all healthcare staff: a national evaluation of Schwartz rounds. J Interprof Care 34(1):140-142. https://doi.org/10. 1080/13561820.2019.1636008

37. Sexton JB, Adair KC, Profit J, Bae J, Rehder KJ, Gosselin T et al (2021) Safety culture and workforce well-being associations with positive leadership walkrounds. Jt Comm J Qual Patient Saf 47(7):403-411. https://doi.org/10.1016/j.jcjq. 2021.04.001

38. Weigl M, Hornung S, Angerer P, Siegrist J, Glaser J (2013) The effects of improving hospital physicians working conditions on patient care: a prospective, controlled intervention study. BMCHealth Serv Res 13(1):401. https://doi.org/10.1186/1472-696313-401

39. Schneider A, Wehler M, Weigl M (2019) Effects of work conditions on provider mental well-being 
and quality of care: a mixed-methods intervention study in the emergency department. BMC Emerg Med 19(1):1. https://doi.org/10.1186/s12873018-0218-x

40. Hooper JJ, Saulsman L, Hall T, Waters F (2021) Addressing the psychological impact of COVID-19 on healthcare workers: learning from a systematic review of early interventions for frontline responders. BMJ Open 11(5):e44134. https://doi.org/10. 1136/bmjopen-2020-044134

41. Pollock A, Campbell P, Cheyne J, Cowie J, Davis B, McCallum J et al (2020) Interventions to support the resilience and mental health of frontline health and social care professionals during and after a disease outbreak, epidemic or pandemic: a mixed methods systematic review. Cochrane Database Syst Rev 11:Cd13779. https://doi.org/10.1002/ 14651858.Cd013779

42. Kisely S, Warren N, McMahon L, Dalais C, Henry I, Siskind D (2020) Occurrence, prevention, and management of the psychological effects of emerging virus outbreaks on healthcare workers: rapid review and meta-analysis. BMJ 369:m1642. https://doi.org/10.1136/bmj.m1642

43. Dewey C, Hingle S, Goelz E, Linzer M (2020) Supporting clinicians during the COVID-19 pandemic. Ann Intern Med 172(11):752-753. https://doi.org/10.7326/m20-1033

44. Strametz R, Raspe M, Ettl B, Huf W, Pitz A (2020) Handlungsempfehlung: Stärkung der Resilienz von Behandelnden und Umgang mit Second Victims im Rahmen der COVID-19-Pandemie zur Sicherung der Leistungsfähigkeit des Gesundheitswesens. Zbl Arbeitsmed 70(6):264-268 https://doi.org/10.1007/s40664-020-00405-7

45. Bourbonnais R, Brisson C, Vezina M (2011) Long-term effects of an intervention on psychosocial work factors among healthcare professionals in a hospital setting. Occup Environ Med68(7):479-486.https://doi.org/10.1136/oem. 2010.055202

46. Aust B, Ducki A (2004) Comprehensive health promotion interventions at the workplace: experiences with health circles in Germany. J Occup Health Psychol 9(3):258-270. https://doi. org/10.1037/1076-8998.9.3.258

47. Teoh KRH, Hassard J, Cox T (2018) Individual and organizational psychosocial predictors of hospital doctors' work-related well-being: a multilevel and moderation perspective. Health Care Manage Rev. https://doi.org/10.1097/hmr.0000000000000207

48. Clay-Williams R, Nosrati H, Cunningham FC, Hillman K, Braithwaite J (2014) Do large-scale hospital- and system-wide interventions improve patient outcomes: a systematic review. BMCHealth Serv Res 14(1):369. https://doi.org/10.1186/14726963-14-369

49. Michie S, West MA (2004) Managing people and performance: an evidence based framework applied to health service organizations. Int J Management Reviews 5-6(2):91-111. https://doi. org/10.1111/j.1460-8545.2004.00098.x

\section{Umweltverschmutzung durch Plastik}

Neuer Arbeitskreis Plastik und Nachhaltigkeit der Deutschen

Dermatologischen Gesellschaft startet Initiative - Vernetzung mit anderen Fachgruppen angestrebt

Der gesamte Gesundheitssektor wird täglich mit Verpackungsmaterialien aus Kunststoffen unterschiedlichster Art geflutet. Im medizinischen Bereich notwendig, um gesetzlich vorgeschriebene Hygienestandards zu erfüllen, stellt die zunehmende Umstellung auf Einmalartikel wie z.B. OP-Besteck und auch die in der Pandemie verwendeten Masken und Kittel ein wachsendes Entsorgungsproblem dar. Dabei ist die Umweltverschmutzung durch Plastik längst zu einer der schwierigsten Herausforderungen unserer Zeit geworden.

Zudem sind Kunststoffe künstlich erschaffene Rohstoffe vielfältigster Art aus Erdöl und Erdgas. Die weltweite Kunststoffproduktion macht aktuell 10 bis $13 \%$ des gesamten Kohlendioxidanteils am Maximalbudget zur Erhaltung des 1,5-Grad-Zieles der Erderwärmung bis 2050 aus. Insgesamt ist der Gesundheitssektor für etwa $5 \%$ der Treibhausgasemissionen verantwortlich und trägt damit aktiv zur Klimakrise bei.

Um die globale Umweltverschmutzung und die Treibhausgase durch Plastik zu reduzieren, muss der Gesundheitssektor auf den Prüfstand gestellt werden. Gerade von dermatologischen Kliniken und Praxen werden regelmäßig Lokaltherapien empfohlen oder verordnet, die möglicherweise gesundheitsschädliche Stoffe enthalten. Winzige Plastikpartikel unterschiedlichster chemischer Zusammensetzung - Mikroplastik und flüssige Polymere - sind zudem biologisch nicht oder nur schwer abbaubar, sie akkumulieren in der Umwelt und gelangen durch die Nahrungskette zurück auf unseren Teller. Anfang 2020 wurde deshalb der Arbeitskreis Plastik und Nachhaltigkeit in der Dermatologie unter dem Dach der Deutschen Dermatologischen Gesellschaft (DDG) gegründet. Der Arbeitskreis verfügt seit April 2021 über eine mehrsprachige Homepage (www.akdermaplastik.de), die relevante Informationen für Mediziner aller Fachrichtungen zu bedenklichen Inhaltsstoffen in Cremes und Pflegeprodukten bündelt. Zudem vernetzt er sich bundesweit mit nachhaltigen Initiativen aus dem Gesundheitssektor, fördert die Bildung ähnlicher Initiativen in anderen medizinischen Fachrichtungen, fokussiert auf Lösungsvorschläge, indem er Qualitätsmanagement (QM)-Vorlagen für die Transformation zur nachhaltigen Praxis erarbeitet und Patienteninformationen zum Download anbietet.

Ärzt*innen aller Fachrichtungen werden aufgerufen, durch die Umsetzung ressourcenschonender Maßnahmen in der Praxis oder Klinik als Multiplikator*innen einen wichtigen Beitrag zur Aufklärung der Bevölkerung, damit zum Umweltschutz und zum Erhalt der Gesundheit eines jeden Individuums zu leisten.

Interessent*innen und Initiativen können sich gerne unter office@akdermaplastik.de melden.

\author{
Arbeitskreis Plastik und Nachhaltigkeit \\ in der Dermatologie (DDG) \\ Dr. med. Dipl. Biol. Susanne Saha (1. \\ Vorsitzende) \\ Fachärztin für Dermatologie \\ Hautärzte am Marktplatz \\ Kaiserstr. 72 \\ 76133 Karlsruhe
}

Quelle: www.akdermplastik.de 
Hier steht eine Anzeige.

黑 Springer 